controlled, add-on trial design. A maximum dosage of $45 \mathrm{mg} / \mathrm{kg} /$ body wt or $3600 \mathrm{mg} /$ day was titrated within 14 days. Felbamate-treated patients had a $34 \%$ decrease in frequency of atonic seizures, as compared with a $9 \%$ decrease in those receiving placebo. The total frequency of seizures was decreased by $19 \%$ in felbamate-treated patients, as compared with a $4 \%$ increase in the placebo group. Side-effects were similar in the two groups. Anorexia, vomiting, and somnolence occurred more frequently in the felbamate group, whereas diarrhea occurred more frequently in the placebo group. (Ritter FJ et al. Efficacy of felbamate in childhood epileptic encephalopathy (Lennox-Gastaut syndrome). N Engl J Med Jan 7 1993; $\underline{328:}$ : 29-33). (Reprints: Dr Frank J Ritter, Minnesota Epilepsy Group, 310 N Smith Ave, Ste 300, St Paul, MN 55102).

COMMENT. Felbamate (2-phenyl-1,3-propanediol dicarbamate) appears to be effective in the treatment of various types of seizures associated with the Lennox-Gastaut syndrome. Side effects are typically mild or moderate in severity and require no change in dosage. According to parental observations, the overall quality of life is also improved by increased alertness and verbal responsiveness.

\title{
NEONATAL ELECTROGRAPHIC SEIZURES
}

Preterm and full-term neonates with electrographically confirmed seizures were compared at the University of Pittsburgh School of Medicine. The incidence of seizures for all neonates admitted to a neonatal ICU was $2.3 \%$. Of 92 neonates with EEG-confirmed seizures seen over a 4 year period, 62 were preterm and 30 were full-term. Seizure frequency of preterm 30 wk neonates was $3.9 \%$ and significantly higher than that of older neonates. Clinical seizures were noted in $45 \%$ of preterm and $53 \%$ of full-term neonates. Seizures were subtle in $71 \%$ and $68 \%$, respectively. Ischemic brain lesions were diagnosed in $39 \%$ of preterm compared to $77 \%$ of full-term neonates. Intraventricular hemorrhage occurred in $45 \%$ of preterm and only in $3 \%$ of full-term neonates. Mortality was higher in the preterm than in the term populations. (Scher MS et al. Electrographic seizures in preterm and full-term neonates: Clinical correlates, associated brain lesions, and risk for neurologic sequelae. Pediatrics Jan 1993; 91: 128-134). (Reprints: Dr Mark S Scher, MageeWomens Hospital, Developmental Neurophysiology Laboratory, 300 Halket St, Pittsburgh, PA 15213).

COMMENT. More than $50 \%$ of this cohort had only electrographic expression of seizures. A later onset of seizures in preterm neonates suggests adverse medical complications after birth. One cannot always rely on simultaneous electrographic confirmation of seizures since $16 \%$ of patients exhibit electroclinical dissociation. 